\title{
Bockdalek Hernia With Gastric Volvulus With Ischemia in An Adult: A Case Report
}

\author{
Saptarshi Biswas ${ }^{\mathrm{a}, \mathrm{c}}$, Patrick McNerney $^{\mathrm{a}}$, Prem Patel $^{\mathrm{b}}$
}

\begin{abstract}
We present an interesting case of a 73-year-old female who presented to the emergency department (ED) with complaints of abdominal pain for the past few days. Patient admitted history of "stomach problems" for the past few years with similar attacks every $3-4$ months and attributed this to her "gallbladder". After her dinner the night before, she started experiencing intense abdominal pain, which prompted her to come to the ED for evaluation. A portable chest X-ray was acquired which showed herniation of the gas-distended stomach overlying the left lung base. CT scan with IV contrast was then performed which confirmed a left-sided diaphragmatic hernia containing the antrum of the dilated stomach but the antrum was twisted to the left of the esophagogastric (EG) junction. There was pneumatosis in the wall of the caudal aspect of the gastric body, suggestive of ischemia. The patient underwent emergent exploratory laparotomy, derotation, partial gastrectomy and gastrostomy where she was found to have an adult Bockdalek hernia on the left side. We discuss this rare case and review relevant literature.
\end{abstract}

Keywords: Bockdalek hernia; Gastric volvulus; Ischemia

\section{Introduction}

Congenital hernias are a relatively uncommon pathology noted in pediatric patients [1]. Bockdalek hernia results from a posterolateral defect caused by incomplete muscular formation of the diaphragm in the region of fusion of the pleuroperitoneal membrane and the septum transversum [2]. The incidence is reported as 1 in 2,200 to 12,500 live births and is more prevalent on the left side as the right pleuroperitoneal fold fuses earlier than left and liver is supposed to act as a barrier [3]. In

Manuscript accepted for publication March 22, 2017

aDepartment of Trauma and Acute Care Surgery, Forbes Hospital, Allegheny Health Network, PA, USA

b Department of Trauma and General Surgery, Westchester University Medical Center, Valhalla, NY, USA

${ }^{\mathrm{c} C}$ Corresponding Author: Saptarshi Biswas, Department of Trauma and Acute Care Surgery, Forbes Hospital, Allegheny Health Network, PA, USA.

Email: saptarshibiswas@comcast.net

doi: https://doi.org/10.14740/jcs315w most instances, there is no peritoneal sac.

Although uncommon, adults can present with complications of Bockdalek hernia such as gastric volvulus, strangulation or incarceration [4].

We report an interesting case in which an adult patient with a transdiaphragmatic hernia presented to the emergency department (ED) and was found to have an adult Bockdalek hernia with gastric volvulus resulting in partial ischemia.

\section{Case Report}

A 73-year-old female with past history of hypertension (HTN) and type 2 diabetes mellitus (DM) presented to the ED with complaints of abdominal pain for the past few days. Patient admitted history of "stomach problems" for the past few years with similar attacks every $3-4$ months and attributed this to her "gallbladder". After her dinner the night before, she started experiencing intense abdominal pain, which prompted her to come to the ED for evaluation.

On presentation, patient complained of abdominal pain, malaise, and poor appetite. Patient stated she had been having normal bowel movements and denied any nausea or vomiting. Review of rest of the systems was essentially negative.

The patient had a past history of gastroesophageal reflux disease (GERD) that had been managed by an $\mathrm{H} 2$ blocker. The other comorbidities included DM II, hypothyroidism, HTN, and depression. Her surgical history comprised of bilateral knee replacements with strep infections and prolonged antibiotic use. Her current medications included atenolol-chlorthalidone, escitalopram, levothyroxine, metformin, and ranitidine. Patient was allergic to penicillin and sulfa drugs.

On physical examination, her vitals were recorded as BP $188 / 86 \mathrm{~mm} \mathrm{Hg}$, pulse 84 , temperature ( $\mathrm{Src}) 36.7^{\circ} \mathrm{C}$ (oral), respiratory rate 21 , and $\mathrm{SpO}_{2} 91 \%$. The abdomen on examination appeared doughy, tender to deep palpation in bilateral upper quadrant, mildly distended, with no rebound or guarding, no masses, and no organomegaly. Rest of the exam was benign. Patient's lab values were: WBC $16,600 / \mu \mathrm{L}$, hemoglobin 11.9 $\mathrm{g} / \mathrm{dL}$, hematocrit $39.2 \%$, and platelets $254,000 / \mu \mathrm{L}$.

Patient underwent right upper quadrants ultrasound which showed a cyst with a mildly thickened septation in the right lobe of the liver measuring $1.4 \times 1.7 \times 1.8 \mathrm{~cm}$. The liver was otherwise unremarkable. The gall bladder was normal with no intra- or extrahepatic biliary dilatation, with the common duct 


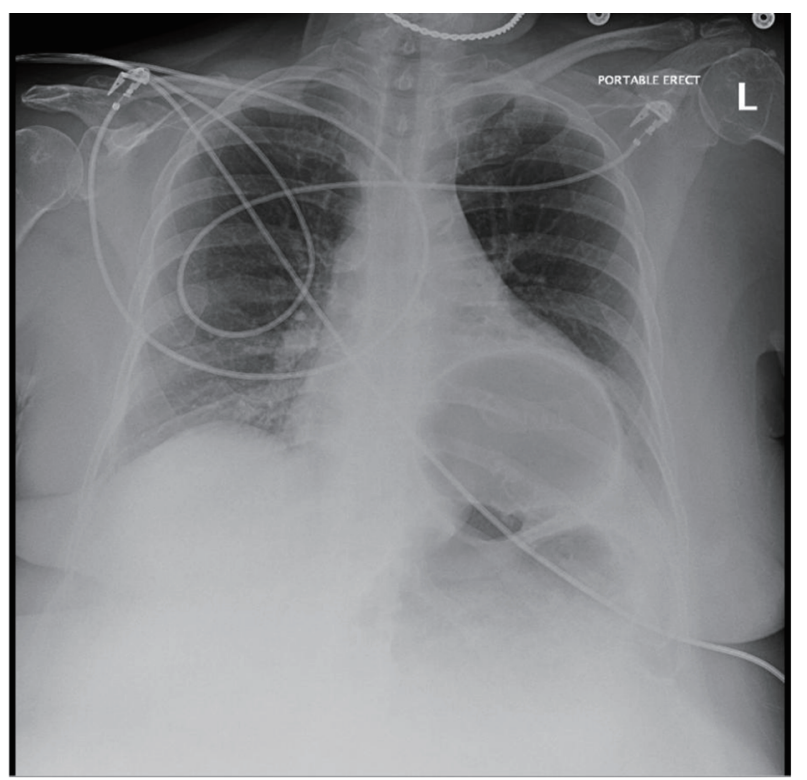

Figure 1. Chest $\mathrm{X}$-ray showing herniation of the gas-distended stomach overlying the left lung base along with some left basilar atelectasis.

\section{measuring $4 \mathrm{~mm}$.}

A portable chest X-ray was acquired in the ED which showed herniation of the gas-distended stomach overlying the left lung base along with some left basilar atelectasis (Fig. 1). CT scan with IV contrast was then performed which confirmed a left-sided diaphragmatic hernia containing the antrum of the dilated stomach. The EG junction was below the diaphragm but the antrum was twisted to the left of the EG junction. There was pneumatosis in the wall of the caudal aspect of the gastric body, suggestive of ischemia (Fig. 2).

\section{Operative details}

An upper midline laparotomy was made sharply with the scalpel, followed by the Bovie electrocautery and tension was used to separate the adipose tissue and maintain the midline. The incision was deepened to the fascia which was grasped with Kocher's elevated and divided sharply. Bovie electrocautery

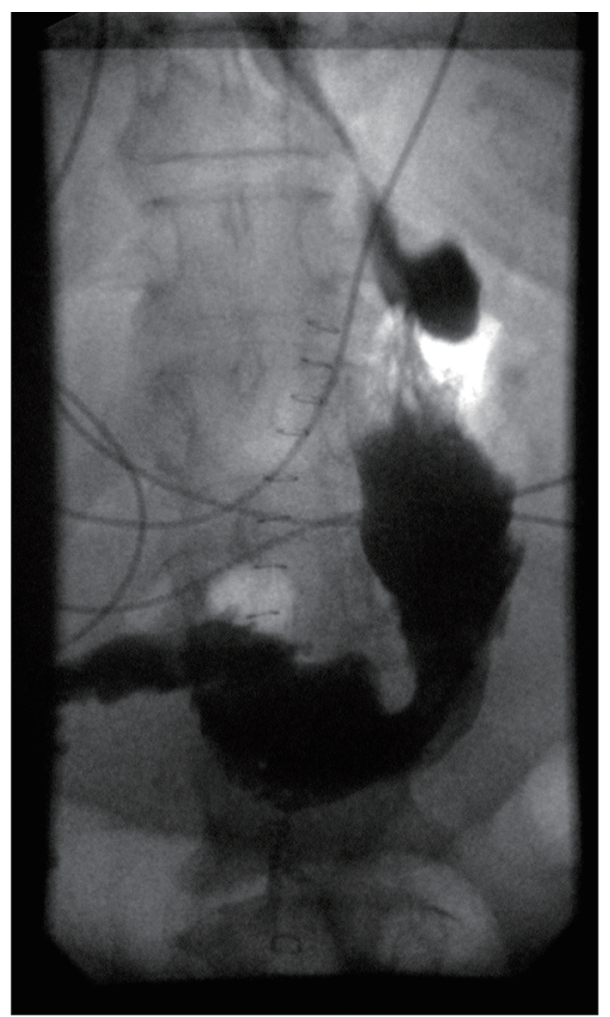

Figure 3. UGI follow through showing no postoperative leak.

was used to divide the fascia under direct visualization while protecting the intra-abdominal contents. The peritoneum was divided with Metzenbaum scissors and then divided in like fashion with the Bovie. Upon entering the peritoneal cavity, there was little room to work and the omentum had migrated superiorly onto the stomach. The incision was extended to just below the umbilicus for additional working room. The omentum was densely adherent as well. It was elevated and divided with the Ligasure Impact to create a window to access and visualize the stomach. The visible stomach was controlled and entered with the Bovie for decompression. After extending the defect sufficient to access the gastric contents. The gastric contents were evacuated removing fluid and generous quantities of undigested or partially digested food. Successful decom-
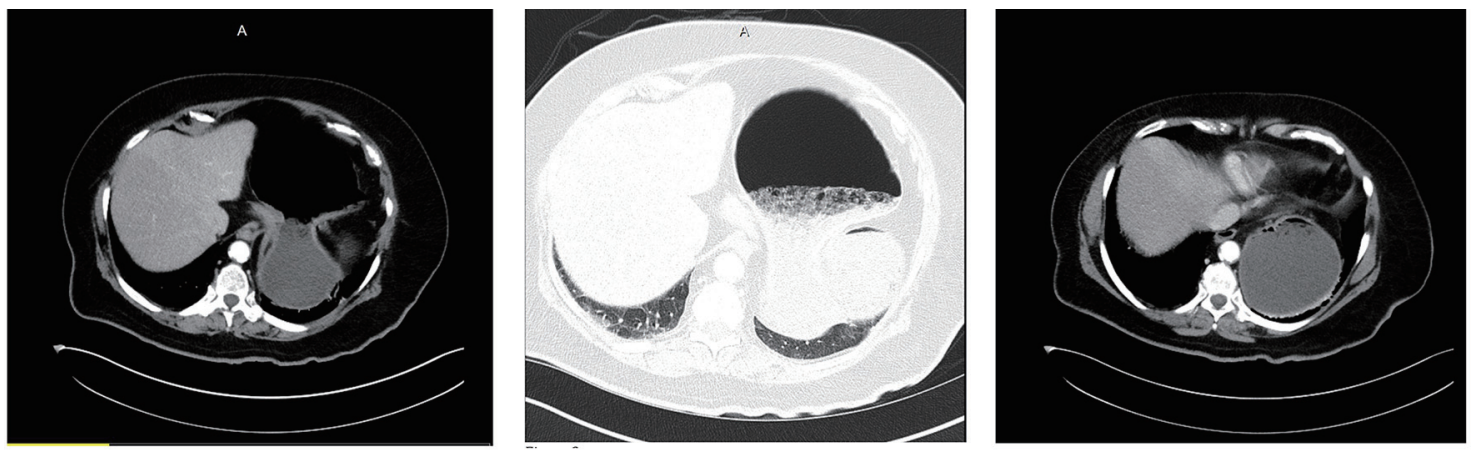

Figure 2. CT scan showing left-sided diaphragmatic hernia with herniation of the antrum and mesentero-axial volvulus. Pneumatosis of the stomach is suggestive of ischemia. 
pression of the contents allowed delivery of the stomach from the thoracic cavity into the abdomen. After gastric lavage and evacuation of contents, the defect was oversewn in two layers of running 3-0 vicryl.

Attention was directed to untwisting the stomach. There were adhesions to the omentum, spleen and adhesive bands to the diaphragm and the diaphragmatic defect, which was separate from the esophageal hiatus. After freeing the adjacent adhesions, the stomach was unfurled, stay sutures were placed for retraction at the most anterior point of the defect, and the hernial content was taken down in its entirety using a combination blunt dissection and Bovie cautery. Adequate hemostasis was ensured and the diaphragmatic defect was completely closed with multiple figure-of-eight 0 proline sutures.

The remaining stomach was quite large, patulous and atonic with necrotic areas near the cardia and adjacent ischemic areas along the greater curve. Ligasure was used to take down the gastro-colic ligament beginning toward to the distal mid body and was carried up to division of the short gastric vessels. Next, five firings of the GIA 80 green were used to remove the necrotic and ischemic portions as well as restore a roughly normal appearing stomach volume. The point of entry for decompression of the stomach was on the posterior aspect of the stomach after it was restored to normal anatomic position. This segment was included in the specimen. There was bleeding from the staple line in multiple places which was controlled with Bovie electrocautery and running locking 3-0 silk. The entire staple line was then dunked and imbricated with interrupted 3-0 silk with care taken to ensure that staple line corners were well covered. All remaining stomach appeared viable.

A gastroscope was introduced into the patient's mouth under direct visualization and passed into the esophagus and stomach. There were no lesions noted in the esophagus. The gastric wall had no bleeding, no ulcers and no remaining ischemic areas. The peritoneal cavity was filled with warm saline and immersed without evidence of leaking air bubbles. Additional retained food was removed via the gastroscope. The pylorus was patent without lesions. Under insufflation, areas for additional reinforcement were identified with placement of additional sutures. The stomach was desufflated and the scope was withdrawn.

To ensure no additional abnormalities, duodenum and gallbladder were examined. There were no gallstones, no encroaching fat, and the liver appeared healthy without signs of cirrhosis or abnormalities. The bowel was then examined from the ligament of Treitz to the fold of Treves followed by the colon down to the rectum and peritoneal reflection. There was sigmoid diverticulosis without evidence of diverticulitis.

A location in the mid body of the stomach was identified to correspond with a proper gastromy site location on the anterior abdominal wall. A 20 French MIC balloon gastrostomy tube was placed through the abdominal wall and stomach after ensuring balloon integrity. The balloon was inflated to $10 \mathrm{~mL}$ and the external washer secured with 3-0 Nylon to the skin. The pursestring sutures were cinched and tied and the abdominal wall sutures were also tied. An NG was passed down and position was confirmed intra-abdominally.

The midline incision was closed in layers and the skin was stapled. The patient tolerated the procedure well and transferred to the PACU in stable condition extubated. Pathology showed stomach specimen with perforation, serositis, extensive areas of ischemic necrosis and vascular congestion. No $H$. pylori or dysplasia was identified.

Patient underwent a UGI follow through on postoperative day 3 (Fig. 3), which showed no evidence of stricture, distortion, or postoperative leak. On postoperative day 5, the patient was discharged home with oral analgesics and stool softener. The patient presented to the outpatient follow-up in the clinic in 2 weeks and has thus far made an unremarkable recovery.

\section{Discussion}

Congenital or acquired defects are responsible for herniation of the abdominal contents into the thorax. Diaphragmatic hernias are commonly produced in the esophageal (hiatal hernia) or paraesophageal hiatus (paraesophageal hernia). Congenital hernias occur through the pleuroperitoneal canal of Bockdalek (posterolateral) or Canal of Morgagni (anteromedial) [2]. Traumatic or postoperative hernias may occur anywhere in the diaphragm [5].

Bockdalek foramen is an opening in the postero-lateral costal part of the fetal diaphragm, typically between ribs 10 and 11 , usually $2-3 \mathrm{~cm}$ in dimension, which communicates between the pleural and peritoneal cavities [6]. Professor Victor Alexander Bockdalek in 1948 first described the failure of the closure. In adults Bockdalek hernias are usually asymptomatic. If symptomatic they commonly present with abdominal or thoracic pain, respiratory distress and bowel obstruction [6]. Gedik et al [7] reported severe symptoms in as high as $46 \%$ of cases with a $32 \%$ mortality due to visceral strangulation or sudden death mostly attributed to intrathoracic complications.

The commonly present organs in Bockdalek hernia are greater omentum, transverse colon, liver, small intestine, and the spleen. Rarely pancreas, appendix, ovary and the Meckels diverticulum are found as hernia contents [8]. Left part of the transverse colon, splenic flexure and the descending colon usually herniate along with the greater omentum [9].

Greater curvature of the stomach can be dragged into the thoracic cavity especially in large defects. The presence of a diaphragmatic defect may predispose to volvulus of the stomach because in congenital diaphragmatic hernia gastrosplenic and gastrophrenic ligaments are elongated or absent [10]. Occasionally the gastric volvulus in the Bockdalek hernia can include the pancreatic tail resulting in pancreatitis [11]. There have been case reports of pancreatitis caused by traction following acute distention of the stomach and subsequent volvulus [12].

Sources of this hiatal distension vary with the most common being secondary gastric volvulus which are most often associated with iatrogenic defects in the diaphragm, typically resulting from surgeries concerning hiatal hernia repair (e.g. Nissen and Allison techniques) [13-15]. Other procedures that are less commonly associated with gastric volvulus include left nephrectomy, splenectomy, and esophogastrectomy [16].

A volvulus is defined as an abnormal twisting of viscera 
around itself or other structures [16]. Gastric volvulus occur relatively infrequently compared to those found in other locations and their pathophysiology is also unique by comparison [16]. Defined as a twisting of the stomach resulting in a closed loop obstruction, a gastric volvulus typically requires torsion of $180^{\circ}$ for such an obstruction. Most typically this rotation occurs longitudinally, often described as an organoaxial torsion [17]. Torsions can also occur along the mesenteroaxial axis and a combination torsion, occurring along both axis, can occur as well [17]. The most common cause of gastric volvulus is hiatal hernia while ligamentous laxity is the main predisposing factor.

Based on etiology, gastric volvulus can be classified into primary or secondary. Primary gastric volvulus occurs due to laxity of the supporting ligaments, thus allowing the stomach to twist along its mesentery [18]. Secondary volvulus can occur due to abdominal adhesions, diaphragmatic eventration, splenic abnormalities, phrenic nerve palsy, traumatic diaphragmatic rupture or even an intrinsic gastric pathology, e.g. tumors acting as the lead point $[18,19]$. Primary gastric volvulus can be treated endoscopically by decompression and derotation of the stomach followed by posterior fixation via percutaneous endoscopic gastrostomy $[19,20]$. This method has also been employed in cases of secondary gastric volvulus but is typically reserved for patients deemed too unstable for surgical intervention [13]. Secondary gastric volvulus are preferred to be treated surgically $[13,21]$. Gastric volvulus can be further classified as acute or chronic. The acute form can cause more than $180^{\circ}$ rotation of the stomach [22] resulting in closed loop obstruction, progressive gastric dilatation and eventual ischemia. If not diagnosed in time, this can lead to infarction and necrosis resulting in rupture and peritonitis. Such complication can have a mortality rate of $42-56 \%$ [18]. However due to rich vascular supply of the stomach severe ischemia only occurs in $5-28 \%$ of cases [19].

Gastric volvulus poses a unique risk to physicians, due to their relative rarity and potentially life-threatening complications, so a high index of suspicion must be maintained when a patient presents with the classical symptoms of intractable vomiting, persistent epigastric pain, and inability/difficulty inserting a nasogastric tube $[23,24]$. This is the classic Bortchardt's triad. Other common symptoms include chest pain, especially postprandial pain, hemorrhage, anemia, inability to belch, fullness, and abdominal distension [25]. As with other volvulus, strangulation and ischemia may possible occur. Acute gastric volvulus have a higher risk of ischemia as they are typically associated with a torsion of over $180^{\circ}$, while chronic volvulus typically present with lower ischemic risk and lower severity of symptoms.

Diagnosis can often be delayed because of non-specific presentations. Chest X-ray findings include retrocardiac air due to intrathoracic portion of the stomach along with mediastinal air fluid levels in some cases. A barium study can help in confirming the diagnosis and also the type of volvulus. It may also determine the degree of obstruction. Although in the acute emergency settings gastrograffin and plain film may be preferred for screening. Negative results may result from inability of the contrast to enter the herniated viscus or when solid organ, e.g. spleen is involved [26]. CT scan is relatively quick and provides a comprehensive detail of the thoracic lesion including the vitality of the stomach [27]. A distended and obstructed stomach can lead to ischemia and perforation which if missed can potentially be fatal. Thoracoscopy, laparoscopy, pneumoperitoneography, pleurography, Liver-Spleen scan and ultrasonography are the other available diagnostic tools, although seldom used in emergency scenarios [28]. Laboratory values are typically non-specific and non-diagnostic.

Surgery typically involves a derotation and reduction of the herniated contents back into the abdominal cavity as well as repair of the defect that allowed for herniation. Fixation of stomach via suture or PEG gastropexy has been shown to reduce recurrence rates [13]. A laparotomy or a laparoscopic approach can be taken although literature leans towards laparoscopic repair. Both thoracic and abdominal approaches have been advocated. The abdominal approach is preferred due to the potential gastrointestinal complications caused by the hernia that can be better repaired through the abdominal approach [29].

\section{Conclusion}

Gastric volvulus results from torsion of all or part of the stomach around one of its axis. This rotation usually occurs on a longitudinal axis within the thoracic cavity. Hiatal hernia is the commonest cause of gastric volvulus. Typically iatrogenic causes, e.g. Nissen and Allison repairs of hiatal hernias need to be considered. Bockdalek hernia result from a failure of closure of the posterior costal diaphragm and attachment of gastric ligaments to the diaphragm resulting in a defect with an increased risk for gastric volvulus. It is usually rare in adults. Gastric volvulus is a diagnostic challenge and therapeutic emergency.

Diagnosis is based on clinical suspicion and radiologic imaging, typically chest X-ray and chest $\mathrm{CT}$. Due to the rarity and high potential for morbidity and mortality, a high index of suspicion is warranted as the consequences of a delayed or missed diagnosis can be potentially fatal. Treatment is surgery and usually laparoscopic approach is preferred (an open laparotomy was performed in our case). Both thoracic and abdominal approach may be tried although the trend favors an abdominal route. The use of mesh is controversial and lacks long-term outcome data but can be used for larger defects. However, smaller defects especially if tension free can be closed primarily.

\section{Conflicts of Interest}

The authors declare that there are no conflicts of interests regarding the publication of this paper.

\section{References}

1. Atef M, Emna T. Bochdalek Hernia With Gastric Volvulus in an Adult: Common Symptoms for an Original Diagnosis. Medicine (Baltimore). 2015;94(51):e2197. 
2. Sugg WL, Roper CL, Carlsson E. Incarcerated Bochdalek Hernias in the Adult. Ann Surg. 1964;160:847-851.

3. Shin MS, Mulligan SA, Baxley WA, Ho KJ. Bochdalek hernia of diaphragm in the adult. Diagnosis by computed tomography. Chest. 1987;92(6):1098-1101.

4. Harrington DK, Curran FT, Morgan I, Yiu P. Congenital Bochdalek hernia presenting with acute pancreatitis in an adult. J Thorac Cardiovasc Surg. 2008;135(6):13961397.

5. Bryer JV, Hegarty MM, Howe C, Rubin D, Angorn IB. Traumatic diaphragmatic hernia. Br J Surg. 1978;65(2):69-73.

6. Granier V, Coche E, Hantson P, et al. Intrathoracic caecal perforation presenting as dyspnea. Case Rep Med. 2010;2010:1-4.

7. Gedik E, Tuncer MC, Onat S, Avci A, Tacyildiz I, Bac B. A review of Morgagni and Bochdalek hernias in adults. Folia Morphol (Warsz). 2011;70(1):5-12.

8. Carter RE, Waterston DJ, Aberdeen E. Hernia and eventration of the diaphragm in childhood. Lancet. 1962;1(7231):656-659.

9. Hoffman E. Strangulated diaphragmatic hernia. Thorax. 1968;23(5):541-549.

10. Ayala JA, Naik-Mathuria B, Olutoye OO. Delayed presentation of congenital diaphragmatic hernia manifesting as combined-type acute gastric volvulus: a case report and review of the literature. J Pediatr Surg. 2008;43(3):E3539.

11. Cuschieri RJ, Wilson WA. Incarcerated Bochdalek hernia presenting as acute pancreatitis. $\mathrm{Br} \mathrm{J}$ Surg. 1981;68(9):669.

12. Butler N, Claireaux AE. Congenital diaphragmatic hernia as a cause of perinatal mortality. Lancet. 1962;1(7231):659-663.

13. Axon PR, Whatling PJ, Dwerryhouse S, Forrester-Wood CP. Strangulated iatrogenic diaphragmatic hernia: a late diagnosed complication. Eur J Cardiothorac Surg. 1995;9(11):664-666.

14. Alrabeeah A, Giacomantonio M, Gillis DA. Paraesophageal hernia after Nissen fundoplication: a real complication in pediatric patients. J Pediatr Surg. 1988;23(8):766768.

15. Testini M, Vacca A, Lissidini G, Di Venere B, Gurrado
A, Loizzi M. Acute intrathoracic gastric volvulus from a diaphragmatic hernia after left splenopancreatectomy: Report of a case. Surg Today. 2006;36(11):981-984.

16. Kim HH, Park SJ, Park MI, Moon W. Acute Intrathoracic Gastric Volvulus due to Diaphragmatic Hernia: A Rare Emergency Easily Overlooked. Case Rep Gastroenterol. 2011;5(2):272-277.

17. Channer LT, Squires GT, Price PD. Laparoscopic repair of gastric volvulus. JSLS. 2000;4(3):225-230.

18. Chau B, Dufel S. Gastric volvulus. Emerg Med J. 2007;24(6):446-447.

19. Sevcik WE, Steiner IP. Acute gastric volvulus: case report and review of the literature. CJEM. 1999;1(3):200-203.

20. Rashid F, Thangarajah T, Mulvey D, Larvin M, Iftikhar SY. A review article on gastric volvulus: a challenge to diagnosis and management. Int J Surg. 2010;8(1):18-24.

21. Takanami I. Hernia of the diaphragm with gastric ulcer and volvulus: an unusual complication after diaphragmatic resection by VATS. Interact Cardiovasc Thorac Surg. 2003;2(4):544-546.

22. Peterson CM, Anderson JS, Hara AK, Carenza JW, Menias CO. Volvulus of the gastrointestinal tract: appearances at multimodality imaging. Radiographics. 2009;29(5):1281-1293.

23. Borchardt M. Zur pathologie und therapie des magen volvulus. Arch Klin Chir. 1904;74:243-260.

24. Kotobi H, Auber F, Otta E, Meyer N, Audry G, Helardot PG. Acute mesenteroaxial gastric volvulus and congenital diaphragmatic hernia. Pediatr Surg Int. 2005;21(8):674676.

25. Porter DN, Emerson C, Ward P. The best way to a woman's heart? Emerg Med J. 2011;28(3):247-248.

26. Johnson DG, Deaner RM, Koop CE. Diaphragmatic hernia in infancy: factors affecting the mortality rate. Surgery. 1967;62(6):1082-1091.

27. Heiberg E, Wolverson MK, Hurd RN, Jagannadharao B, Sundaram M. CT recognition of traumatic rupture of the diaphragm. AJR Am J Roentgenol. 1980;135(2):369-372.

28. Adamthwaite DN. Diaphragmatic hernia presenting itself as a surgical emergency. Injury. 1984;15(6):367-369.

29. Shah R, Sabanathan S, Mearns AJ, Choudhury AK. Traumatic rupture of diaphragm. Ann Thorac Surg. 1995;60(5):1444-1449. 